\title{
COMMENTARY
}

\section{Parenteral omega-3 fatty acids: pouring oil on troubled waters?}

\author{
Philip C Calder* \\ See related research by Pradelli et al., http://ccforum.com/content/16/5/R184
}

\begin{abstract}
A meta-analysis of parenteral fish oil in 23 studies in intensive care unit (ICU) and non-ICU patients reported a reduced infection rate (significant in ICU patients) and shorter lengths of ICU and hospital stays (both non-ICU and ICU patients). Parenteral fish oil reduced inflammation and improved oxygenation index and liver function. The findings of the meta-analysis are discussed in this report.
\end{abstract}

In the previous issue of Critical Care, Pradelli and colleagues [1] presented a new meta-analysis of parenterally administered marine omega- 3 fatty acids (also known as 'fish oil') in non-intensive care unit (non-ICU) (elective surgical) and ICU patients, with results considered separately and in combination. An earlier metaanalysis restricted to patients undergoing elective major abdominal surgery concluded that parenteral fish oil significantly reduces infection rate (odds ratio: 0.56; $P=0.04$ ), length of ICU stay (stay 1.92 days shorter; $P=0.004$ ), and length of hospital stay (stay 2.98 days shorter; $P=0.0005$ ) [2]. Pradelli and colleagues [1] extend this earlier analysis by considering a broader range of patients, by including papers published between 1997 and 2011, and by reporting on findings for a range of laboratory parameters in addition to the clinical outcomes. Ultimately, 23 studies were included (13 of patients admitted to the ICU and 10 of patients undergoing major abdominal surgery and not admitted to the ICU), involving a total of 1,502 patients. Of the studies involving the ICU, nine were of patients who had undergone major abdominal surgery. The other four studies involved patients with sepsis [3], critical illness [4], acute respiratory

\footnotetext{
*Correspondence: pcc@soton.ac.uk

Human Development and Health Academic Unit, Faculty of Medicine, University of Southampton, IDS Building, MP887 Southampton General Hospital, Tremona Road, Southampton, SO16 6YD, UK
}

distress syndrome [5], or severe acute pancreatitis [6]. There was no effect of parenteral fish oil on mortality, either overall ( $\mathrm{n}=10$ studies) or in the two separate patient groups. Furthermore, there was no effect on bleeding-related outcomes, although these were reported in relatively few studies. However, the analysis confirmed that, for the non-ICU and ICU patients combined, parenteral fish oil significantly reduces infection rate ( $\mathrm{n}=11$ studies published from 1997 to 2011; risk ratio: $0.61 ; P=0.002)$, length of ICU stay $(\mathrm{n}=8$ studies published from 1997 to 2010; stay 1.92 days shorter; $P=0.005)$, and length of hospital stay $(\mathrm{n}=14$ studies published from 1997 to 2011; stay 3.29 days shorter; $P=0.0005$ ) [2]. The similarity of the findings to those of the earlier, apparently more restricted, meta-analysis by Chen and colleagues [2] is striking. The reasons for this similarity are that there is significant overlap of papers considered in the two analyses and that, although the study by Chen and colleagues was restricted to patients undergoing abdominal surgery, those authors combined findings for non-ICU and ICU patients. In the new metaanalysis, parenteral fish oil significantly reduced infection rate in non-ICU but not in ICU patients, although the sizes of the effect were fairly similar in the two groups (risk ratios were 0.53 in non-ICU patients and 0.71 in ICU patients). In contrast, the effects on hospital length of stay were significant in both groups of patients $(-1.86$ days in non-ICU patients and -5.17 days in ICU patients). Lung oxygenation index was reported in two studies in septic ICU patients $[3,6]$ and overall was found to be significantly improved with parenteral fish oil.

The findings of this meta-analysis are highly encouraging as far as patients in the ICU are concerned. There are two possible explanations for the lack of a significant effect on infection rate in ICU patients despite significant reductions in ICU and hospital stays. The first explanation is that there is no immune effect of fish oil (and therefore no effect on infection rate) and that the benefits on length of stay relate to other effects (for example, on inflammation [7] or gas exchange [3,6] or both). The second explanation is that there is an immune effect of fish oil, resulting in shorter or less severe infections - but 
not in changes in the infection rate (that is, in the rate of becoming infected) - and this translates into a more rapid recovery. Although parenteral fish oil significantly reduced length of hospital stay in non-ICU patients, many of these patients would not normally receive parenteral nutrition and certainly not for the typical period of 5 days used in the included studies. Furthermore, the lengths of hospital stay reported in these studies in non-ICU patients have little relevance to reallife clinical care of most patients undergoing uncomplicated elective abdominal surgery.

Pradelli and colleagues [1] present, for the first time, a meta-analysis of laboratory outcomes. Of relevance to inflammation, parenteral fish oil was found to significantly decrease interleukin-6 levels and to significantly increase the ratio of omega-3 eicosapentaenoic acid (EPA)-derived leukotriene (LT) $\mathrm{B}_{5}$ to omega-6 arachidonic acid (ARA)-derived $\mathrm{LTB}_{4}$ with similar effects in ICU and non-ICU patients. The significance of the latter finding is that $\mathrm{LTB}_{4}$ is a potent chemoattractant for leukocytes and plays a role in amplifying lung inflammation but that $\mathrm{LTB}_{5}$ is much weaker in this regard [8]. Thus, this effect on fatty acid-derived mediators may be linked directly to the reported significant improvement on oxygenation index in ICU patients, which in turn may be linked to shorter lengths of ICU and hospital stays. The production of the LTs is influenced by the relative availability of the substrate fatty acids, EPA and ARA. EPA is the major omega-3 fatty acid in fish oil, and when fish oil is administered parenterally, EPA appears in greater amounts in blood lipids [3,6,9] and in immune cells [10]. Liver enzymes were significantly decreased by parenteral fish oil in both ICU and non-ICU patients, indicating an improvement in liver viability.

Overall, this meta-analysis confirms the potential for improved cell and tissue function and clinical outcomes with parenteral fish oil in both ICU and non-ICU patients. Nevertheless, the individual studies considered have typically been small and often have not individually reported the improved outcomes seen with metaanalysis. Future research should focus on larger trials in patients in the ICU and should aim to consolidate the clinical findings and to establish the causal chain of events from increased omega- 3 fatty acid status through to improved clinical outcome and the optimal dose of fish oil required to exert the benefits seen.

\section{Abbreviations}

ARA, arachidonic acid; EPA, eicosapentaenoic acid; ICU, intensive care unit; LP, leukotriene.

\section{Competing interests}

The author serves on the Scientific Advisory Boards of the Danone Research Centre for Specialised Nutrition (Wageningen, The Netherlands) and Pronova Biocare (Lysaker, Norway) and has received speaking honoraria from Fresenius Kabi (Bad Homburg, Germany), B. Braun (Melsungen, Germany), Abbott Nutrition (Abbott Park, IL, USA), and Baxter Healthcare (Deerfield, IL, USA).

Published: 12 November 2012

\section{References}

1. Pradelli L, Mayer K, Muscaritoli M, Heller A: N-3 fatty acid-enriched parenteral nutrition regimens in elective surgical and ICU patients: a meta-analysis. Crit Care 2012, 16:R184.

2. Chen B, Zhou Y, Yang P, Wan HW, WU XT: Safety and efficacy of fish oilenriched parenteral nutrition regimen on postoperative patients undergoing major abdominal surgery: a meta-analysis of randomized controlled trials. JPEN J Parenter Ent Nutr 2010, 34:387-394.

3. Barbosa VM, Miles EA, Calhau C, Lafuente E, Calder PC: Effects of a fish oil containing lipid emulsion on plasma phospholipid fatty acid, inflammatory markers, and clinical outcomes in septic patients: a randomized, controlled clinical trial. Crit Care 2010, 14:R5.

4. Friesecke S, Lotze C, Kohler J, Heinrich A, Felix SB, Abel P: Fish oil supplementation in the parenteral nutrition of critically ill medical patients: a randomized controlled trial. Intensive Care Med 2008, 34:1411-1420

5. Sabater J, Masclans JR, Sacanell J, Chacon P, Sabin P, Planas M: Effects of an omega-3 fatty acid-enriched lipid emulsion on eicosanoid synthesis in acute respiratory distress syndrome (ARDS): a prospective, randomized, double-blind, parallel group study. Nutr Metab (Lond) 2011, 8:22.

6. Wang X, Li W, Zhang F, Pan L, Li N, Li J: Fish oil-supplemented parenteral nutrition in severe acute pancreatitis patients and effects on immune function and infectious risk: a randomized controlled trial. Inflammation 2009, 32:304-309.

7. Calder PC: The 2008 ESPEN Sir David Cuthbertson Lecture: Fatty acids and inflammation - from the membrane to the nucleus and from the laboratory bench to the clinic. Clin Nutr 2010, 29:5-12.

8. Calder PC: Fatty acids and inflammation: the cutting edge between food and pharma. Eur J Pharmacol 2011, 668:S50-58.

9. Mayer K, Fegbeutel C, Hattar K, Sibelius U, Krämer HJ, Heuer KU, TemmesfeldWollbrück B, Gokorsch S, Grimminger F, Seeger W: Omega-3 vs. omega-6 lipid emulsions exert differential influence on neutrophils in septic shock patients: impact on plasma fatty acids and lipid mediator generation. Intensive Care Med 2003, 29:1472-1481.

10. Versleijen MW, Roelofs HM Rombouts C, Hermans PW, Noakes PS, Calder PC, Wanten GJ: Short-term infusion of a fish oil-based lipid emulsion modulates fatty acid status, but not immune function or (anti)oxidant balance: a randomized cross-over study. Eur J Clin Invest 2012, 42:290-302.

\section{doi:10.1186/cc11848}

Cite this article as: Calder PC: Parenteral omega- 3 fatty acids: pouring oil on troubled waters? Critical Care 2012, 16:172. 